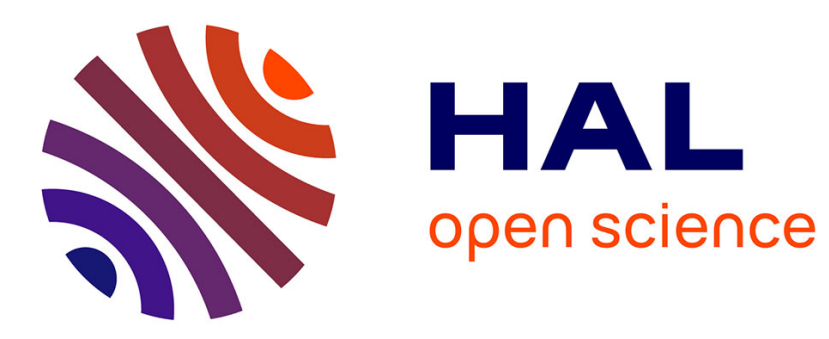

\title{
Security of the Medical Media Using an Hybrid and Multiple Watermark Technique
}

Mohamed Kallel, M Bouhlel, Jean Lapayre

\section{To cite this version:}

Mohamed Kallel, M Bouhlel, Jean Lapayre. Security of the Medical Media Using an Hybrid and Multiple Watermark Technique. IJIG, International Journal of Image and Graphics, 2011, 11 (1), pp.103-115. hal-01222907

\section{HAL Id: hal-01222907 https://hal.science/hal-01222907}

Submitted on 30 Oct 2015

HAL is a multi-disciplinary open access archive for the deposit and dissemination of scientific research documents, whether they are published or not. The documents may come from teaching and research institutions in France or abroad, or from public or private research centers.
L'archive ouverte pluridisciplinaire HAL, est destinée au dépôt et à la diffusion de documents scientifiques de niveau recherche, publiés ou non, émanant des établissements d'enseignement et de recherche français ou étrangers, des laboratoires publics ou privés. 


\title{
SECURITY OF THE MEDICAL MEDIA USING AN HYBRID AND MULTIPLE WATERMARK TECHNIQUE
}

\author{
MOHAMED KALLEL \\ University Sciences and Technologies of Image and Telecommunications. Higher Institute of Biotechnology, \\ Sfax-TUNISIA \\ kallel_mohamed2004@yahoo.fr
}

MOHAMED SALIM BOUHLEL

University Sciences and Technologies of Image and Telecommunications. Higher Institute of Biotechnology, Sfax-TUNISIA

medsalim.bouhlel@enis.rnu.tn

JEAN-CHRISTOPHE LAPAYRE

Informatics laboratory of Franche Comte LIFC (FRE CNRS 2661) Besançon - France

jean-christophe.lapayre@univ-fcomte.fr

Received Day Month Year

Revised Day Month Year

\begin{abstract}
This paper proposes a new approach of multiple and hybrid watermark using two linked fields of insertion. This approach associates two different series of marks: one is sturdy guaranteeing the supervision of diffused media though the network and testifying to the identity of the owner of the medical image; the other series of marks, which is frail, insuring the integrity, the trace and the archives of medical diagnoses. In fact, this approach allows to benefit from the advantages of different insertion spaces (spatial and frequency field) and kind of two different fields of watermark. In the spatial field, the diagnosis of the doctor (frail marks) is inserted guaranteeing its records and its trace. In the frequency, the sturdy mark is inserted allowing to ensure the automatic control of the media through the network and testifying to the proprietor of the medical image.
\end{abstract}

Keywords: Multiple watermark, hybrid watermark, spatial field, frequency field, security, integrity, trace.

\section{Introduction}

The rapid expansion of the internet and the development of digital technologies have increased the accessibility of digital media. For this reason, the image authentication becomes very important. Therefore, the security techniques are required where verification of integrity [Fourati et al (2008)] and guaranteeing record and trace of each intervenient. The use of the watermark technique in medical field has many benefits. In fact, the watermark technique can be used to guarantee the trace of the doctor's diagnoses. Then, it follows the evolutionary state of the patient and it forms a whole report of the patient. Hybrid and multiple watermark technique provides a possible 
solution to the above problem. In fact, in this sheet of paper, we are going to conceive a new approach of multiple and hybrid watermark using two linked fields of insertion. In section 1, we are going to explain the choice of the two insertions field. In the next section, we are going to give the positive effect of our approach and we are going to explain the insertion phase and the extraction phase. Then we are going to provide an optimism technique to increase the sturdiness of our approach. Finally, we will present experimental results in section 4. Conclusions are drawn in section 5.

\section{The choice of frequency/spatial field}

This approach associates two different series of marks: one is sturdy guaranteeing the supervision of diffused media though the network and testifying to the identity of the owner of the medical image; the other series of marks, which is frail, insuring the integrity, the trace and the archives of medical diagnoses.

The spatial field provides an important capacity of insertion [Stankovié et al (2001)] and arranges a higher image quality than other insertion field [Kallel et al (2007)]. In fact this author used in this work a spatial field to ensure the imperceptibility of the mark and to guarantee the information integrity. For this reason, we have chosen the spatial field and we are going to be limited to only four combinations which are the following: frequency/spatial, spatial/frequency, spatial/wavelet, wavelet/spatial.

It is noted that the combination spatial/wavelet, wavelet/spatial are to be eliminated because the issued space of waved decomposition is considered as a spatial field [Trichili et al (2002)]. In fact, this author has proved that the spatial field and the wavelet field was considered the same because the tow field can specify the place of the pixel in the image (spatial localization). Thus, the spaces are limited only to two combinations. As our

multiple watermark application is vulnerable to any eventual transformation (frail watermark). So, we are going to apply it in the second insertion field. As a consequence, we are going to study the hybrid watermark in the frequency/spatial field.

\section{The positive effect of our approach}

Just as for every watermark method, our approach is formed of insertion and extraction phase which we are going to explain in detail for every insertion field. On the one hand, in the medical field, the information is authentic and very important and we must ensure a secure transfer of this file. For this reason, we have judged that it is imperative to use the spatial field for the application of our approach guaranteeing in this way, high image quality, enough insertion capacity in order to introduce the diagnoses of different doctors and integrity of the inserted mark in view of the fact that the LSB plan insertion offers a certificate of authenticity during the extraction phase [Giakoumaki et al (2003)]. On the other hand, it will be important to use the frequency field for the sturdy mark insertion allowing to ensure the automatic control of diffused media through the network and testifying to the proprietor of the medical image. The presence of this sturdy mark prevents any attempt of pirate taking advantage then of this insertion field. 


\section{The insertion phase}

\subsection{The insertion phase in the frequency field}

In this phase, we have set for objective the duality between sturdiness in comparison to different attacks and the imperceptibility of the mark.

\subsubsection{Choice of porters coefficients of the mark}

The insertion procedure in the DCT (Discrete Coefficient Transform) field is too delicate because a little modification of low frequencies may cause a strong degradation of the quality of the image. As a result, the insertion in high frequencies may proved to be inefficient since a lesser attack which the image may undergo, can also change the inserted mark. That's why; it is very interesting to choose a tape of average frequencies insuring a good compromise between high resistance towards different attacks and a low degradation of the image quality. Consequently, we have chosen the average frequencies for the insertion of our mark.

\subsubsection{Insertion diagram}

In the frequency domain, the amount of asked calculation, which performs a transformation on a whole image, is important. That's why; we divide our image with size $256 \times 256$ pixels in 1024 blocks with size $8 \times 8$ pixels; and every block will be tattooed [EMEK and. Pazarc1 (2005)].

We apply for every block the DCT; every bit of the chosen mark will be inserted in a coefficient belonging to the tape of average frequencies under a substitutive way according to the following insertion formula:

$$
y_{i}=b_{i}+\alpha
$$

With

$$
\begin{aligned}
& y_{i} \text { : coefficient of the porter image of the mark } \\
& b_{i}: \text { bit of the inserted mark }
\end{aligned}
$$

The parameter $\alpha$ is used to clarify the degree of perceptibility of the mark. It will be used as a factor of compromise between a good imperceptibility and an efficient sturdiness. We have chosen to insert the binary acronym of the European project $\mathrm{TeNeCi}$ (TeleNeurology Cooperative) with size of 32x32 bits as shown in figure 1 .

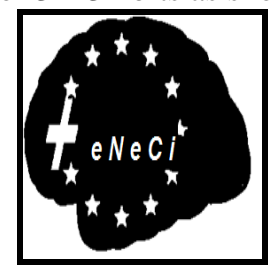

Fig. 1. The project acronym

The inverse DCT is eventually applied following the insertion of mark in order to rebuild the watermarked image. 


\subsection{The insertion phase in the spatial field}

The insertion phase in the spatial field consists of converting the original image into a binary code. Then, the carried out diagnosis via the doctor is converted into ASCII code next in a binary code in order to insert in the zone to which it was permitted in the coded original image with the help of a reserved key [Kallel et al (2007)]. This procedure is repeated successively for every concerned doctor so as to achieve a multiple watermark technique [Osborne et al (2004)].

\section{The extraction phase}

\subsection{The extraction phase in the frequency field}

This phase allows to check whether the inserted mark during the insertion phase is present or not in a watermarked image. In our diagram, we have insured this checking without having resort to the original image, only the inserted mark is necessary. The extraction diagram which we have developed focuses on a successive inverse operations than those adopted during the insertion phase. So, we are going to cut up the image into 1024 blocks with $8 \times 8$ pixels. To every block, we apply the DCT and we separate the porter coefficient of the mark.

Following the extraction of the mark, we move to the identification step which aims at proving the source of the image and guarantee its characteristics. For this reason, we have created a bank of 999 marks which are generated randomly to which we add our original mark. Next, we calculate the correlation between the extracted mark and the thousand of marks of the bank. As to confirm that the detection of the mark is efficient, it is compulsory that the maximum correlation suits to the inserted mark.

This correlation is defined as the following:

$$
\mathrm{Cor}=\langle\mathrm{A}, \mathrm{B}\rangle=\frac{\sum_{m} \sum_{n}\left(A_{m n}-\bar{A}\right)\left(B_{m n}-\bar{B}\right)}{\sqrt{\left(\sum_{m} \sum_{n}\left(A_{m n}-\bar{A}\right)^{2}\right)\left(\sum_{m} \sum_{n}\left(B_{m n}-\bar{B}\right)^{2}\right)}}
$$

A and B are respectively the inserted mark and the extracted one.

With: $\bar{A}$ the average of the coefficient of the A matrix.

$\bar{B}$ the average of the coefficient of the B matrix.

\subsection{Extraction phase in the spatial field}

In this extraction phase of the spatial field, we perform the conversion into a binary code of the watermarked image in order to have a binary image composed of 8 plans. Then, we 
extract the mark and we transform it to ASCII code. Next into a character chain so as to have eventually the carried out diagnosis on be half of the correspondent doctor(s).

\section{Optimism}

For our approach of watermark, the first inserted mark must be indeed sturdy as well since, this concealed mark in the medium, is the guarantor of the identity of its right. Once it erased, transformed or substituted, the image is no longer protected and its owner do not possess any proof of copyright as well. Besides, the user of this image is not certain on behalf of its servant.

So we have judged important to increase the sturdiness of our approach; we have had then the idea of inserting every bit of the mark many times in the coefficient of every block of the modified image (in the average frequencies of the frequency field). This redundancy has offered us more chance of finding the inserted mark during the extraction phase. In every block, we are going to insert one bit of the binary mark with its size $32 \times 32$ bits with a redundant manner. In fact, the same bit will be inserted 3 times in 3 different coefficients of tape of average frequencies for every block of the transformed image. This redundancy has offered us during the extraction phase more chance in order to find the exact value of the inserted bit guaranteeing then a well-sturdiness of the develop approach.

Therefore, we have inserted an important amount of information, which lifts the major constraint in the domain of image watermark which is the compromise between the sturdiness of the system and the quality of the watermarked image. In fact, when we insert the information with a redundant matter, the system becomes sturdier but in counterpart, it damages more the medical image.

In addition, we are going to measure the correlation within and without the suggested optimization and this in order to quantify the fidelity of the extracted image contrary to the inserted mark. The figure 2 presents the variation of the correlation for the different tested images.

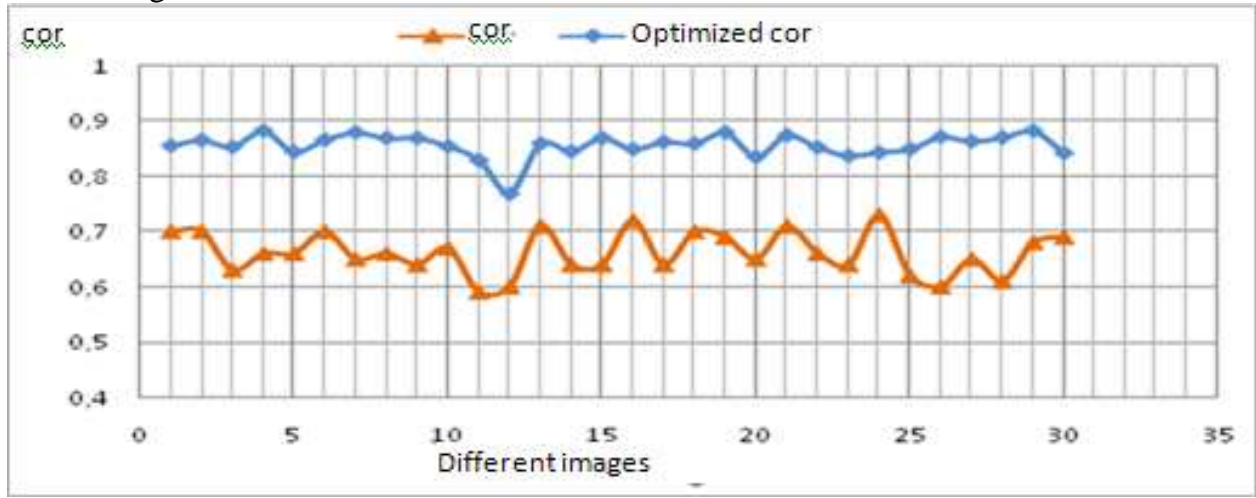

Fig. 2. The correlation variation 
According to the above figure, we notice that the variation of the correlation for the optimistic approach presents high value in comparison to classical elaborate approach. This is done, in fact, to the redundant insertion of the same mark in the image increasing then the possibility to get back it during the extraction phase.

The figure 3 presents the variation of PSNR before and after the optimization and this is so to measure the degradation which was undergone by the image following the insertion of the mark [Fourati et al (2008)].

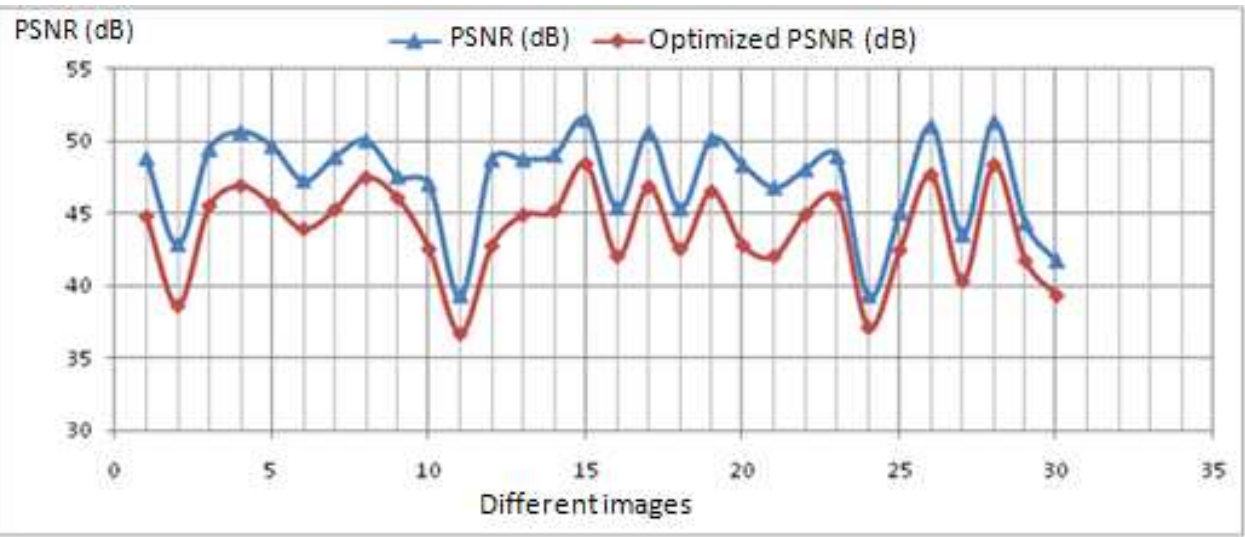

Fig. 3. PSNR variation

We note, according to this figure, that the optimistic approach presents a lower PSNR variation than the classical one. But, it always keeps satisfactory values as they are superior to $30 \mathrm{~dB}$ [Puech and Rodrigues (2004)].

To sum-up, we can notice that for the optimistic approach, the correlation increases whereas the PSNR decreases. This is expected as the increasing of the amount of the inserted information leads to the quality of the original image less better and more accentuated correlation.

\section{Results}

We apply this technique to 30 different medical images with the size of $256 \times 256$ pixels and $8 \mathrm{bit} /$ pixels resolution in order to evaluate the watermark image, to check the sturdiness of the first inserted mark in the frequency domain and the sensibility of the second insertion mark in the spatial field in comparison to different attacks and more precisely the capability of the approach of guaranteeing the trace and the records of the inserted diagnoses.

\subsection{The quality of the watermarked image}

The imperceptibility of the inserted mark is primordial mainly for the medical images as the least modification of the medical image may lead to inappropriate diagnoses. Figure 4 shows the imperceptibility of the image following the insertion in the two fields. 


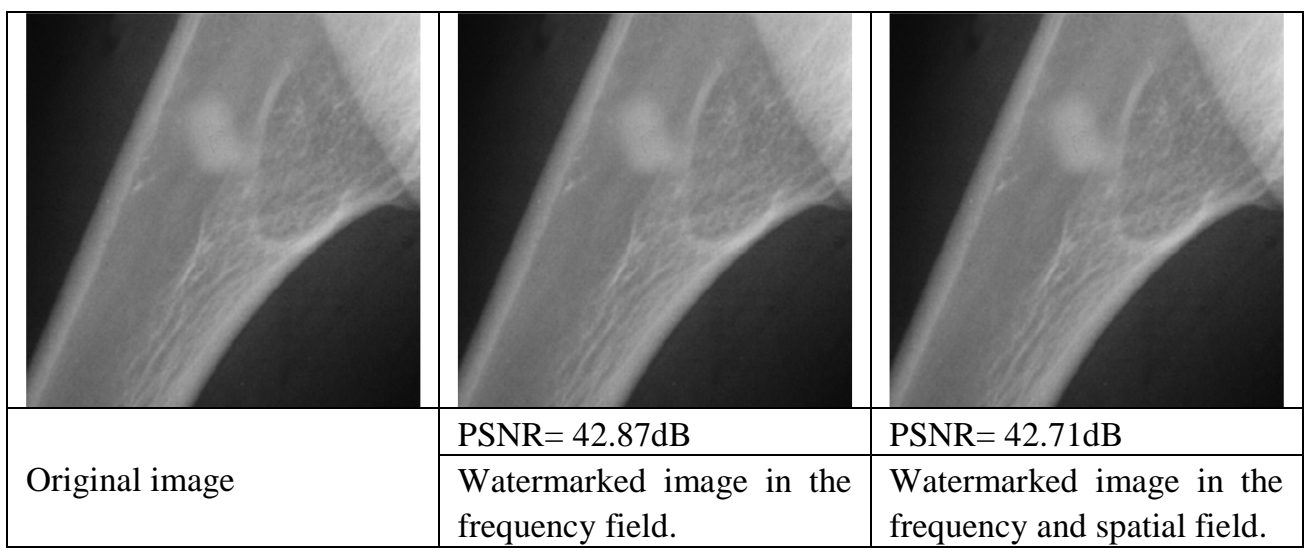

Fig. 4. The quality of the image following its insertion in the two fields

According to the figure 4, we can notice that following the insertion in the frequency field, the watermarked image presents a PSNR of $42,87 \mathrm{~dB}$; which proves the imperceptibility of the first series marks. After the insertion of the diagnoses in the spatial field, the final watermarked image presents a PSNR of 42,71 dB; and eventually the good quality of this medical image.

\subsection{The sturdiness of the first mark in comparison to different attacks}

In order to prove the sturdiness of the developed approach in the frequency field in comparison to different attacks, we have tried to apply possible diverse attacks which the image can undergo as it is shown in table 1. We have used the JPEG compression, added noise, histogram equalization, and filter.

Table1. Sensibility in comparison to attacks following the insertion in the frequency filed

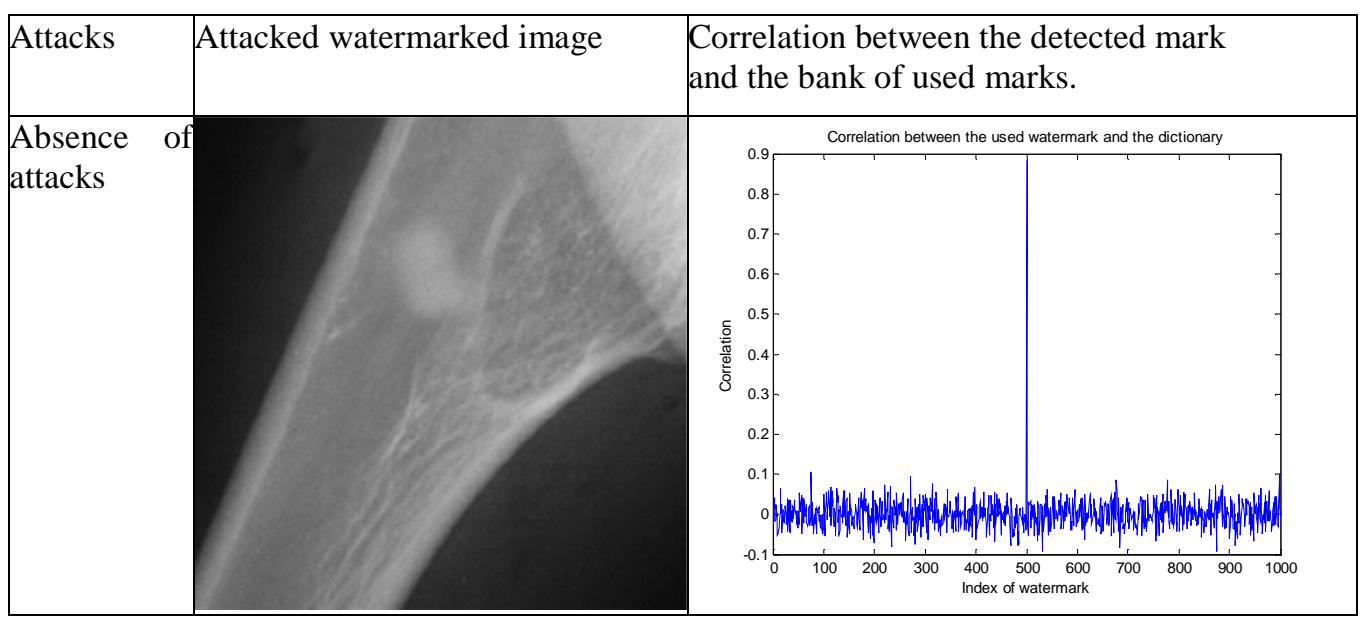


8 Mohamed Kallel et al

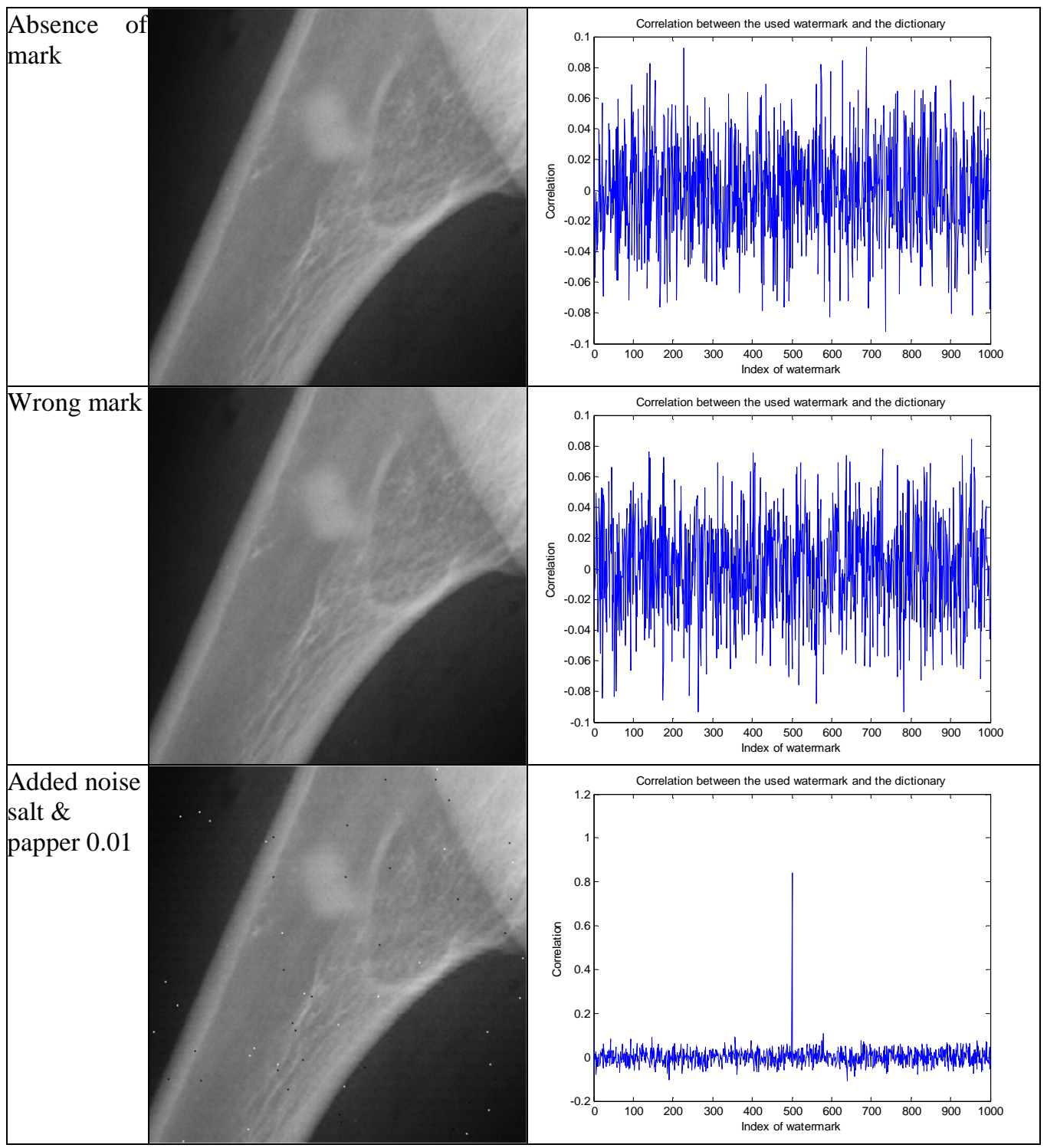


mustn't detect the mark if nothing is inserted in the image. The obtained curve, in this case, doesn't present any peak (and thus no detected mark), which proves the efficiency of our approach that allows to distinguish the case where no watermark is presented in the image. Moreover, the developed watermark approaches permits to deny incorrect marks. We have inserted in the image another binary mark with the size of $32 \times 32$ pixels as it is mentioned in figure 5

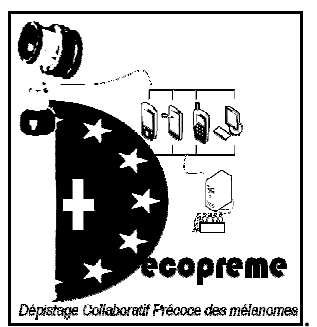

Fig. 5. Incorrect mark

We have tried to detect the mark of this image in order to ensure the efficiency of our approach and prove that it distinguishes from the inserted mark and doesn't detect wrong marks. The curve of the finding correlation in this case (table1) doesn't present any peak, hence the efficiency of this approach and its ability to distinguish the inserted mark one from another.

\subsection{The sensibility of the second set of marks in comparison to different attacks}

In table 2, we are going to study the sensibility of the watermark diagram in comparison to different types of attacks.

Table2. Sensibility in comparison to attacks during the insertion in the fields

\begin{tabular}{|c|c|c|c|}
\hline & Inserted message: pathology & $\begin{array}{l}\text { Extracted message } \\
\text { fréq/spatial }\end{array}$ \\
\hline \multirow{5}{*}{ 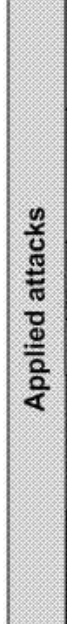 } & 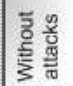 & $\mathbf{x}$ & pathology \\
\hline & 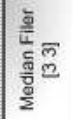 & $\mathbf{x}$ & okidsujgfe \\
\hline & 总宫 & $\mathbf{x}$ & qe'sxpmg \\
\hline & 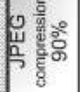 & $\mathbf{x}$ & jolkhgfwc \\
\hline & 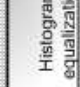 & $\mathbf{x}$ & a' 'rf yhgf \\
\hline
\end{tabular}


With the reference to this table, we notice that the ultimate attacks, which the medical image can undergo, cause the non-comprehensibility of the inserted mark. In fact, The JPEG compression, the histogram equalization, the median filter and the added noise causing the modification in the image character. The used watermark being frail, the modification which have changed the image, reflect also on the set of the inserted marks. For the case of non-attacked image (intact and non falsified image), the inserted diagnosis is well-detected: character by character and this for all the set of inserted marks by the different interventionists guaranteeing then the record of the diagnoses and the trace of the doctors.

Once, the image underwent an attack during its transmission, the vulnerable inserted diagnosis in the extraction phase is incomprehensible so as to warn the user of the presence of the change of the transmitted image avoiding then every risk of having a wrong diagnostic or a different diagnostic from the one inserted.

\section{Conclusion}

In this paper, we have presented a multiple and hybrid watermark approach using both two different fields of insertion. We have inserted in the frequency field a sturdy mark allowing to guarantee the automatic control of the diffused media through the network and testify to the identity of the proprietor of the medical image, the presence of this sturdy mark prevents any attempts of pirate. In the spatial field, we have included the diagnoses of the doctors (frail marks) guaranteeing its records and its trace. We have explained in this research the choice of the two fields of the used insertions by clarifying its advantages. Then, we have explained in detail the insertion and the extraction phase in the two aforementioned fields. Next, we have studied the performance of our approach according to different criteria: the sturdiness of the first mark, the quality of the watermarked image, the sensibility of the second mark (the series of diagnoses) in comparison to different attacks and the guarantee of the trace and the archive of diagnoses. We have also optimized the developed approach by increasing the criterion of sturdiness through a multiple insertion of the same sturdy mark. This redundancy offers us, in fact, more possibility in order to find the inserted mark in the first field of insertion following the extraction phase.

This developed technique is used so as to help the doctors, in a collaborative work, to take the decision in a short time. 


\section{References}

Fourati Kallel I, Bouhlel M S, Lapayre J-C and Garcia E (2008) "Control of Dermatology Image Integrity using Reversible Watermarking"IJIST International Journal of Imaging Systems and Technology, 19: 5-9.

Stankovié S., Djurovié I and Pitas I, (2001) "Watermarking in the Space/Spatial-Frequency Domain Using Two-Dimensional Radon-Wigner Distribution”, IEEE transactions on image processing, 10, 650-658.

Trichili H, Boublel M.S, Derbel N.and Kamoun L.,(2002) "A new medical image watermarking scheme for a better telediagnosis", Proceedings of the IEEE International Conference on Systems, Man and Cybernetics 1, 556-559.

Giakoumaki A, Pavlopoulos S, Koutouris D.,(2003) “A medical image watermarking scheme based on wavelet transform", Proceedings of the 25th Annual International Conference of the Engineering in Medicine and Biology Society IEEE, 1,.856- 859.

Emek S.and Pazarcı M., (2005) "A Cascade DWT-DCT Based Digital Watermarking Scheme", 13th. European Signal Processing Conference (EUSIPCO 2005),

Kallel M., Lapayre J.C and Bouhlel M.S (2007)“A multiple Watermarking Scheme for Medical Image in the Spatial Domain", ICGST International Journal on Graphics, Vision and Image Processing (GVIP), 7.

Osborne D. Abbott D, Sorell M, Rogers D.(2004) "Multiple embedding using robust watermarks for wireless medical images". 3thd international conference on mobile and ubiquitous multimedia. MUM 245-250.

Fourati. Kallel I., Lapayre J.C and Bouhlel M.S (2008)"Medical Image semi fragile Watermarking In The Frequential Field" JOTE Journal Of Testing and Evaluation, 36,

Puech W. and Rodrigues J. M (2004).. A New Crypto-Watermarking Method for Medical Images Safe Transfer”. In EUSIPCO'04 - European Signal Processing Conference, 1,1481-1484. 